\title{
Síndrome trófico trigeminal
}

\section{Trigeminal trophic syndrome}

\section{María Lertora ${ }^{1}$, Patricia Della Giovanna ${ }^{2}$, Hugo Néstor Cabrera ${ }^{3}$ y Sandra García ${ }^{4}$}

\begin{tabular}{ll} 
RESUMEN & \\
$\begin{array}{l}\text { El síndrome trófico trigeminal es una enfermedad infrecuente carac- } \\
\text { terizada por úlceras faciales autoprovocadas en regiones de anestesia } \\
\text { o hipoestesia, secundarias al daño del nervio trigémino. Presentamos } \\
\text { tres casos de este síndrome en seguimiento en nuestro centro. }\end{array}$ & $\begin{array}{l}\text { Palabras clave: síndrome trófico trigeminal, úlceras faciales, úlceras } \\
\text { autoprovocadas. }\end{array}$ \\
\hline
\end{tabular}

\begin{tabular}{l} 
ABSTRACT \\
$\begin{array}{l}\text { Trigeminal trophic syndrome is an uncommon disease characterized by } \\
\text { self-inflicted facial ulcers in anesthetic or hypoesthetic areas, secondary } \\
\begin{array}{l}\text { to trigeminal nerve damage. We present three cases in follow up at our } \\
\text { hospital. }\end{array}\end{array} \quad$ ulcers. \\
\hline
\end{tabular}

\footnotetext{
1 Jefa de Residentes del Servicio de Dermatología

2 Jefa del Servicio de Dermatología

${ }^{3}$ Profesor Consulto en Dermatología

${ }^{4}$ Jefa del Servicio de Anatomía Patológica

Hospital Nacional Profesor Alejandro Posadas, El Palomar, Provincia de Buenos Aires, Argentina
}

Contacto del Autor: María Lertora

E-mail: marialertorael@gmail.com

Fecha de trabajo recibido: $26 / 10 / 2020$

Fecha de trabajo aceptado: 18/3/2021

Conflicto de interés: los autores declaran que no existe conflicto de interés.

\section{INTRODUCCIÓN}

El síndrome trófico trigeminal (STT) es una causa infrecuente de úlceras faciales autoprovocadas descripto por Wallenberg en 1901. También conocido como ulceración neurotrófica trigeminal, es desencadenado tras el daño al nervio trigémino, que genera anestesia o disestesia predominante en el área perinasal y ulceración autoinflingida en el ala nasal ${ }^{1,2}$.

\section{SERIE DE CASOS}

\section{Caso clínico 1}

Una paciente de 83 ańos, con antecedente de tratamiento ablativo con alcoholización del ganglio de Gasser por neuralgia del trigémino, consultó por una dermatosis localizada en el ala nasal derecha, caracterizada por una úlcera asociada a hipoalgesia y parestesias. En el interrogatorio, refirió una manipulación continua de la zona. Cabe destacar que entre la ablación del ganglio de Gasser y la aparición de la dermatosis transcurrieron 40 años (Foto 1).

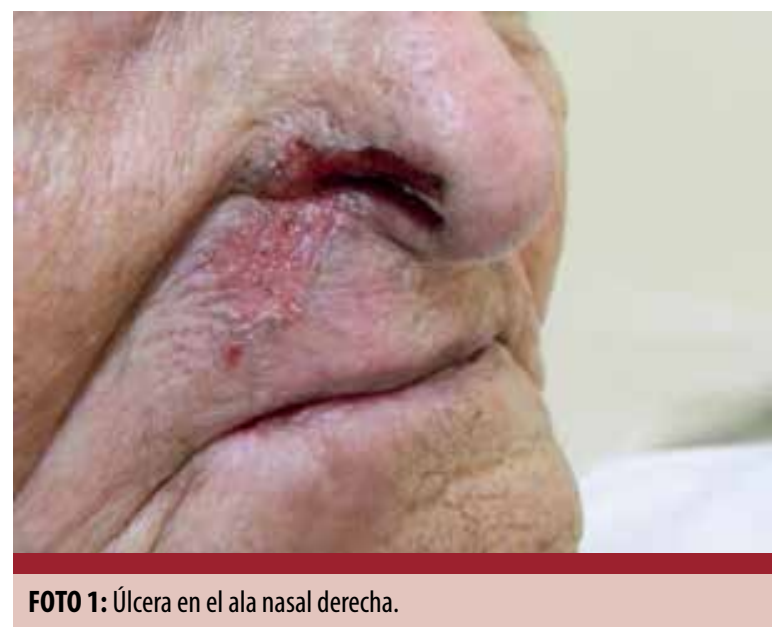




\section{Caso clínico 2}

Una paciente de 60 años consultó por una dermatosis localizada en el ala nasal derecha, caracterizada por una úlcera que apareció después de 6 meses de haber sufrido un accidente cerebrovascular isquémico con hemiplejía faciobraquiocrural derecha. La paciente refería manipular constantemente la zona (Foto 2).

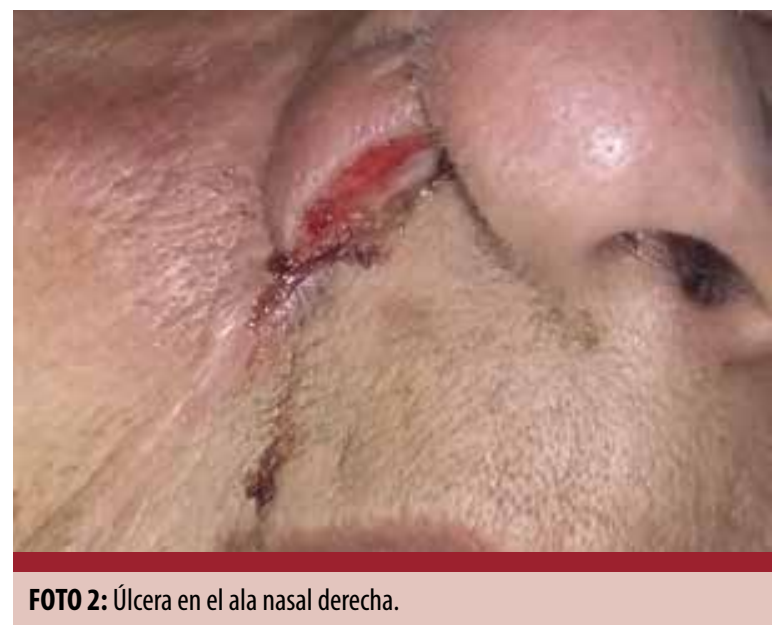

\section{Caso clínico 3}

Una paciente de 59 ańos consultó por la presencia de una dermatosis de 3 años de evolución, ubicada en la piel del cuero cabelludo y caracterizada por úlceras cubiertas por costras. A su vez, se encontraba en seguimiento por el servicio de oftalmología debido a una úlcera en la córnea derecha. La paciente presentaba antecedentes de neuralgia del trigémino del lado derecho, que requirió una intervención quirúrgica (Foto 3).

En las tres pacientes, las biopsias de piel informaron sobre cambios inflamatorios crónicos inespecíficos y se descartaron las causas infecciosas. Los resultados de laboratorio fueron normales y las tomografías computadas del macizo facial no evidenciaron datos positivos.

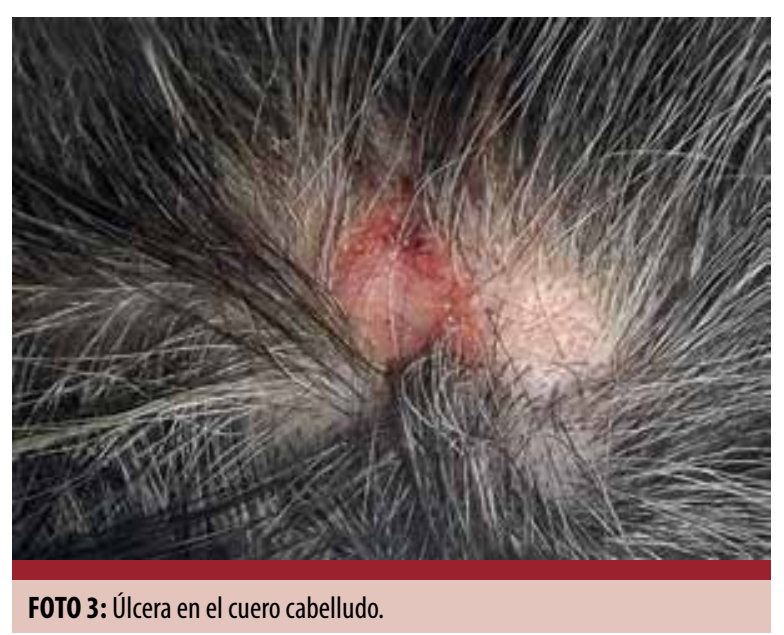

\section{COMENTARIOS}

El STT se describe con una tríada caracterizada por la presencia de una úlcera en el ala nasal asociada a parestesias y anestesia en grado variable, secundaria al daño del nervio trigémino o sus ramas ${ }^{3-5}$.

Afecta a ambos sexos, con un ligero predominio en las mujeres ${ }^{5}$. La edad de presentación varía y es más frecuente en los adultos, con un promedio de edad de 60 años $^{3-5}$. Estos datos concuerdan con las características de los casos presentados en cuanto al sexo y la edad. El período de latencia entre el evento desencadenante y el inicio de la sintomatología puede oscilar desde días hasta décadas, con un promedio de latencia de un año ${ }^{3-5}$. En nuestra experiencia, los casos 2 y 3 tuvieron un período de latencia de 6 meses. Destacamos que la paciente del caso 1 presentó el STT después de 40 años de la ablación del ganglio de Gasser.

Si bien las causas desencadenantes son múltiples, las principales son las iatrogénicas (ablación del nervio trigémino) y los accidentes cerebrovasculares ${ }^{3-5}$. Otras etiologías incluyen cirugía craneofacial, traumatismo, tumores (astrocitomas, meningiomas), infecciones (sífilis, herpes zóster, lepra, varicela), insuficiencia vertebrobasilar, trastornos psiquiátricos, enfermedad de Alzheimer, neuroma del acústico, depósitos amiloides, daño neurológico posparto y uso de marihuana sintética. Es idiopático en un $5 \%$ de los $\operatorname{casos}^{3-9}$.

Clínicamente, se caracteriza por la tríada compuesta por anestesia, parestesias y ulceración del ala nasal, la cual es la localización más frecuente ${ }^{3}$. En ocasiones, se acompaña de compromiso de la frente, mejilla y labio superior homolateral. Otras ubicaciones comprenden labio superior, mejilla, frente, orejas, mandíbula, paladar y cuero cabelludo. Rara vez, puede comprometer los ojos, huesos, cerebro y senos paranasales ${ }^{4}$.

Las úlceras pueden ser únicas o múltiples, en ocasiones comprometen grandes superficies. Si bien suele ser unilateral con mayor afectación del lado derecho, se ha publicado un caso de presentación bilateral ${ }^{4,5}$. Dos de las pacientes tuvieron un cuadro característico con compromiso del ala nasal derecha, mientras que la del caso 3 presentó úlceras en el cuero cabelludo y compromiso de la córnea del lado derecho, por lo que destacamos lo infrecuente de esta localización.

En cuanto a la sintomatología acompañante, la hipoestesia y la anestesia, la sensación de hormigueo, la quemazón, el ardor y el prurito fueron los más referi$\operatorname{dos}^{3,5,7}$.

Con respecto a la fisiopatogenia, el prurito de origen neuropático vinculado al daño nervioso da lugar a un rascado crónico que, como no provoca dolor por la 
anestesia, favorece la formación de úlceras ${ }^{2,5,10}$. Estas persisten por la manipulación repetitiva de la zona, en general durante el sueńo e, incluso, durante la vigilia de modo inconsciente ${ }^{4,10}$.

Aunque los antecedentes neurológicos y el cuadro clínico de ulceraciones unilaterales son claves diagnósticas, se deben realizar estudios histológicos, microbiológicos y de laboratorio para descartar diagnósticos diferenciales como carcinoma basocelular o espinocelular, infecciones, leishmaniasis, histoplasmosis, paracoccidioidomicosis, vasculitis y dermatitis facticia ${ }^{4-6,9,11}$. En el caso de esta última, los estudios de laboratorio son normales y la biopsia de piel descarta otras causas de úlceras faciales. El antecedente neurológico es el factor que puede dividir ambas entidades. Por otra parte, los pacientes con dermatitis facticia suelen padecer una dermatosis bilateral que no respeta la topografía clásica del STT, refieren que el rascado de las lesiones les genera una sensación de alivio y reconocen que el cuadro es autoprovocado ${ }^{4,6,9}$.

En relación con el tratamiento, todavía no se cuenta con un protocolo definido ${ }^{4,5}$. Representa un desafío terapéutico y debe ser abordado por un equipo multidisciplinario con la participación de dermatólogo, oftalmólogo, neurólogo, psicólogo y cirujano ${ }^{4,6,7,11}$. El primer paso, y el más importante, consiste en la educación del paciente y poder transmitirle la característica de la autoprovocación de las lesiones y la importancia de controlar la manipulación de la zona ${ }^{4,5}$.

Como medidas generales, se pueden utilizar medidas oclusivas locales durante el descanso y se aconseja el uso de guantes, además de hacer hincapié en que el paciente tenga las uñas cortas. Las alternativas farmacológicas incluyen gabapentina, carbamazepina, amitriptilina y pregabalina, las cuales pueden combinarse con tacrolimus al $0,1 \%$ local.

Los esquemas propuestos consisten en el uso de gabapentina $200 \mathrm{mg} /$ día combinado con tacrolimus al $0,1 \%$ tópico, carbamazepina $200 \mathrm{mg} 3$ veces por día y amitriptilina $25 \mathrm{mg} 3$ veces por día combinada con diazepam. Los beneficios clínicos con el uso de pregabalina fueron limitados. Las respuestas terapéuticas con estos esquemas se observaron entre el mes y los 6 meses; las recaídas fueron frecuentes ${ }^{1,2}$. También se describió el uso de suplementos de vitamina B y de infiltraciones con triamcinolona. Los esteroides tópicos no fueron eficaces. Entre las alternativas quirúrgicas, se encuentran la estimulación eléctrica transcutánea del nervio, la simpatectomía y, en los pacientes que resuelven el hábito de la autoprovocación de las lesiones, la cirugía reconstructiva ${ }^{1,4-7}$. Se publicó recientemente el caso de un paciente tratado con radiocirugía ${ }^{12}$. Es importante la realización de un seguimiento prolongado 5 .

En nuestra experiencia, las tres pacientes fueron tratadas con curaciones, medidas oclusivas y pregabalina $50 \mathrm{mg} /$ día. La paciente número 1 abandonó el seguimiento luego de indicado el tratamiento y se desconoce su evolución. La paciente 2 presentó el cierre parcial de la úlcera en el ala nasal derecha y abandonó el seguimiento tras un año de tratamiento. En el caso de la tercera paciente, la evolución fue favorable y está en la etapa de epitelización de la úlcera en el cuero cabelludo después de 3 meses de tratamiento (Foto 4). Se encuentra en seguimiento conjunto con los servicios de oftalmología, neurología y psiquiatría. A pesar de haber mejorado, persisten la sensación de hormigueo y el prurito, por lo que por el momento no se plantea la posibilidad de disminuir la dosis de pregabalina. En este caso, la educación de la paciente respecto de su enfermedad fue un pilar en el tratamiento.

El STT es una causa infrecuente de úlceras faciales autoprovocadas y representa un importante desafío diagnóstico y terapéutico. Si bien hasta ahora no se cuenta con protocolos de tratamiento, creemos que con el mayor conocimiento de la entidad será posible optimizar el abordaje y el seguimiento de los pacientes afectados.

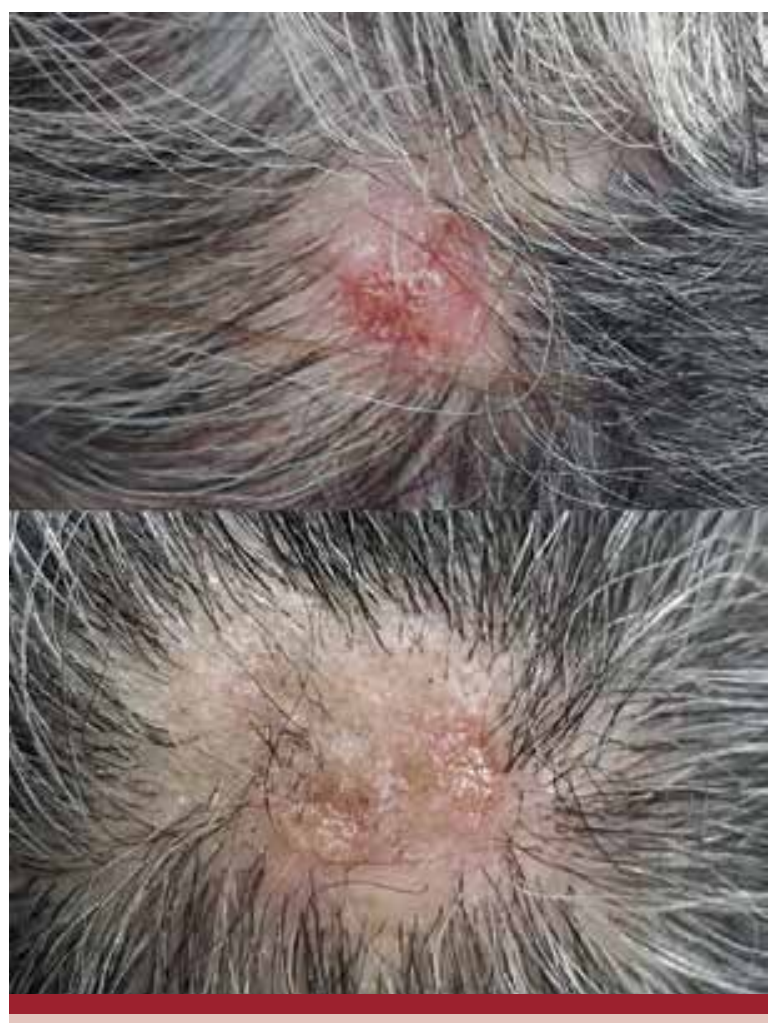

FOTO 4: Evolución clínica favorable, con epitelización de la úlcera en la piel del cuero cabelludo. 


\section{BIBLIOGRAFÍA}

1. Rashid RM, Khachemoune A. Trigeminal trophic syndrome. J Eur Acad Dermatol Venereol 2007;21:725-731.

2. Fernández-Bernáldez A, Rodríguez-Jiménez $P$, HerreroMoyano M, Sánchez-Pérez J. Síndrome trófico trigeminal secundario a recidiva de meningioma. Actas Dermosifiliogr 2020;111:171-173.

3. Garza I. The trigeminal trophic syndrome: an unusual cause of face pain, dysaesthesias, anesthesia and skin/ soft tissue lesions. Cephalalgia 2008;28:980-985.

4. Khan AU, Khachemoune A. Trigeminal trophic syndrome: an updated review. Int J Dermatol 2019;58:530-537.

5. Sawada T, Asai J, Nomiyama T, Masuda K, et ál. Trigeminal trophic syndrome: Report of a case and review of the published work. J Dermatol 2014;41:525-528.

6. Luna P, Nocito M, Cañadas N, Castellanos Posse ML, et ál. Síndrome trófico trigeminal. Una causa infrecuente de úlceras faciales. Arch Argent Dermatol 2009;59:21-23.

7. Sadeghi P, Papay FA, Vidimos AT. Trigeminal trophic syndrome - report of four cases and review of the literature. Dermatol Surg 2004;30:807-812.

8. Khan FA, Manacheril R, Ulep R, Martin JE, et ál. Trigeminal trophic syndrome associated with the use of synthetic marijuana. Ochsner J 2017;17:292-295.

9. Gómez de Castro C, Vázquez-López F, García-García B, Requena López $\mathrm{S}$, et ál. Trigeminal trophic syndrome simulating rodent ulcer basal cell carcinoma: a new clinico-dermoscopic approach. An Bras Dermatol 2017;92:148-150.

10. Oaklander AL. Common neuropathic itch syndromes. Acta Derm Venereol 2012;92:118-125.

11. Zambernardi A, Digilio M, Spiner $R$, Feinsilber D, et ál. Síndrome trófico del trigémino. Un caso con extensión infrecuente. Dermatol Argent 2018;24:57-59.

12. Sallabanda K, Sallabanda M, Barrientos HD, Santaolalla $\mathrm{I}$, et ál. Trigeminal trophic syndrome secondary to refractory trigeminal neuralgia treated with CyberKnife ${ }^{\circledR}$ radiosurgery. Cureus 2020;12:e7670.

\section{DERMATÓLOGOS JÓVENES}

\section{* Elección múltiple: PLACA ERITEMATOVIOLÁCEA EN UN DEDO}

Agostina Alonzo Caldarelli, Pilar Targize Vaquero y Marlene Goland Servicio de Dermatología, HIGA Prof. Dr. Rodolfo Rossi, La Plata, Provincia de Buenos Aires, Argentina

Una mujer de 37 años, sin antecedentes patológicos de relevancia, consultó por la presencia de una lesión en el pulpejo del segundo dedo izquierdo de 8 meses de evolución, asintomática, de comienzo abrupto y tamaño estable, no relacionada con un traumatismo previo, sangrante ante el roce mínimo.

En el examen físico se observaba una placa de color rojo violáceo, de $7 \mathrm{~mm}$ de diámetro mayor, con bordes bien definidos, superficie irregulary una pústula central. No había frémito ni aumento de la temperatura local (Foto 1).

La dermatoscopia reveló un patrón monomorfo compuesto por múltiples lagunas rojo-lechosas, bien delimitadas, de diferentes tamaños, distribuidas en toda la lesión (Foto 2).

\footnotetext{
1) ¿Cuál es su diagnóstico más probable?

a) Hemangioendotelioma retiforme.

b) Angioma.

c) Angioqueratoma solitario.

d) Malformación arteriovenosa digital acral.

e) Hemangioma capilar lobulillar.
}

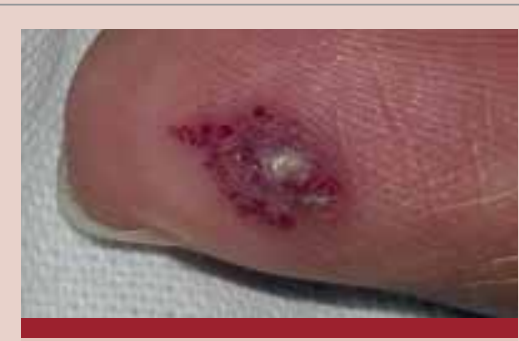

FOTO 1.

2) ¿Qué estudio solicitaría para arribar al diagnóstico definitivo?

a) Ecografía Doppler arterial y venosa.

b) Estudio histopatológico.

c) Radiografía de la mano.

d) Laboratorio con recuento de plaquetas.

e) Las opciones c. y d. son correctas.

3) ¿Qué esperaría encontrar en el estudio histopatológico?

a) Conductos vasculares arborizantes con patrón retiformey células endoteliales en forma de tachuela.

b) Vasos dérmicos entrelazados que forman un ovillo capilar.

c) Vasos subepidérmicos dilatados asociados con acantosis e hiperqueratosis.

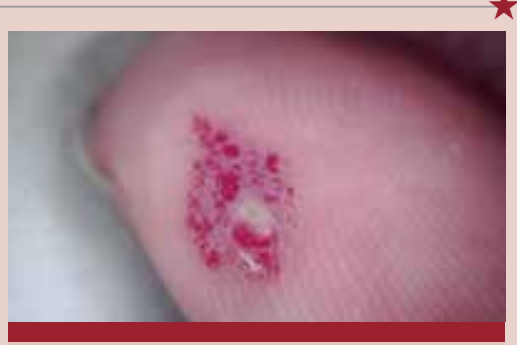

FОTO 2.

d) Múltiples interconexiones entre las arterias de mediano o gran calibre y las venas, sin interposición del lecho capilar.

e) Lobulillos pequeños de capilares dispuestos en una matriz fibromixoide, exofítica y delimitados por un collarete epidérmico hiperplásico.

4) ¿Qué tratamiento consideraría de elección?
a) Extirpación total de la lesión.
b) Láser.
c) Crioterapia.
d) Conducta expectante.
e) Todas las opciones son correctas. 